\title{
Preoperative and intraoperative gastric tumor localization
}

\author{
Kyong Hwa Jun \\ Department of Surgery, St. Vincent's Hospital, College of Medicine, The Catholic University of Korea, Seoul, Korea
}

\begin{abstract}
As the incidence of early gastric cancer increases, gastric tumor localization has become an important issue. Several diagnostic methods have been proposed for preoperative and intraoperative gastric tumor localization. These include endoscopic metal clipping, computed tomographic gastrography, endoscopic tattooing, and intraoperative endoscopy. However, in spite of various methods, tumor localization has its limitations; thus, new diagnostic alternatives need to be developed.
\end{abstract}

Keywords: Stomach neoplasms, Diagnosis, Tumor location

\author{
Received April 19, 2021 \\ Accepted May 5, 2021 \\ Corresponding author \\ Kyong Hwa Jun \\ Department of Surgery, The Catholic \\ University of Korea, St. Vincent's \\ Hospital, 93 Jungbu-daero, Paldal-gu, \\ Suwon 16247 , Korea \\ Tel: $+82-31-881-8636$ \\ Fax: +82-31-547-5347 \\ E-mail: dkkwkh@catholic.ac.kr \\ ORCID: \\ https://orcid.org/0000-0003-3909-5230
}

Copyright $\odot$ The Korean Society of Endoscopic and Laparoscopic Surgeons.
This is an Open Access article distributed under the terms of the Creative Commons Attribution Non-Commercial License (http:// creativecommons.org/licenses/by-nc/4.0/) which permits unrestricted non-commercial use, distribution, and reproduction in any medium, provided the original work is properly cited.
As the incidence of early gastric cancer rises, the need for gastric tumor localization further increases. Furthermore, in the era of minimally invasive surgery, the development of preoperative or intraoperative tumor localization techniques for small-sized tumors has become more important. Under laparoscopic gastric surgery, it is difficult to determine the actual location and safe resection margins of the tumor. Thus, tumor localization is important because laparoscopic surgery has less tactile sensation than open surgery, and with the increase in the early diagnosis of gastric cancer, tumors are smaller.

There are several types of tumor localization methods in gastric cancer surgery. The first is to perform an endoscopy during surgery. However, this increases the operation time, and the air inflation of the intestines makes the operation difficult. The second method is to use a metal clip during the endoscopy and prior to the surgery. With this technique, the patient may be exposed to radiation as an X-ray may be taken to confirm the position of the metal clip during surgery [1]. The third method is endoscopic tattooing which uses tattooing agents such as methylene blue, India ink, and indocyanine green. This could lead to a technical failure due to ineffective tattooing or result in intraperitoneal complications caused by tattoo chemical spillage [2]. All of these techniques require additional endoscopic examination or a portable X-ray or ultrasound.

In this issue of Journal of Minimally Invasive Surgery, Lee et al. [3] report a promising result of intraoperative localization with transpyloric optic navigation (TPON) of the tumor without additional instruments. There may be a risk of tumor dissemination through the opening created, and contamination may occur because a laparoscope that has passed through the stomach is used. However, the authors stated that the risk of cancer dissemination will be low even with saline irrigation of the instrument. The authors introduced the feasibility and safety of TPON while maintaining adequate luminal distension and preventing possible tumor cell seeding by directing the opening toward the extracorporeal area. All the patients were precise in the visibil- 
ity of the localization during surgery and had negative surgical resection margins. The mean proximal margin length was 41.7 $\pm 26.8 \mathrm{~mm}$ and the minimal proximal margin length was $5 \mathrm{~mm}$. In addition, there were no complications related to surgical site infection induced by TPON. This study was a retrospective study comprised of a small sample size, which may have introduced surgeon's selection bias. Despite these limitations, the study demonstrated the efficacy of intraoperative tumor localization with TPON. However, a large-scale prospective study is warranted for standard guidelines regarding effective intraoperative tumor localization.

\section{NOTES}

\section{Conflict of interest}

The author has no conflicts of interest to declare.

\section{REFERENCES}

1. Lee CM, Park S, Park SH, et al. A comparison between two methods for tumor localization during totally laparoscopic distal gastrectomy in patients with gastric cancer. Ann Surg Treat Res 2016;91:112-117.

2. Ushimaru Y, Omori T, Fujiwara Y, et al. The feasibility and safety of preoperative fluorescence marking with indocyanine green (ICG) in laparoscopic gastrectomy for gastric cancer. J Gastrointest Surg 2019;23:468-476.

3. Lee S, Kim GH, Kang DY, Kim DY, Ryu SY. Transpyloric optic navigation of tumor using a laparoscope during totally laparoscopic distal gastrectomy for gastric cancer. J Minim Invasive Surg 2021;24:76-83. 